\title{
BMJ Open Age-related trends in lipid levels: a large-scale cross-sectional study of the general Chinese population
}

\author{
Lei Feng (D) , ${ }^{1}$ Shiyan Nian, ${ }^{2}$ Zongwu Tong, ${ }^{3}$ Ying Zhu, ${ }^{4}$ Ying Li, ${ }^{4}$ Chunting Zhang, ${ }^{4}$ \\ Xuejing Bai, ${ }^{5}$ Xuan Luo, ${ }^{5}$ Mengna Wu, ${ }^{5}$ Zefeng Yan $^{5}$
}

To cite: Feng L, Nian S, Tong Z, et al. Age-related trends in lipid levels: a large-scale crosssectional study of the general Chinese population. BMJ Open 2020;10:e034226. doi:10.1136/ bmjopen-2019-034226

- Prepublication history for this paper is available online To view these files, please visit the journal online (http://dx.doi. org/10.1136/bmjopen-2019034226).

LF and SN contributed equally.

Received 11 September 2019 Revised 14 February 2020 Accepted 19 February 2020

\section{Check for updates}

(c) Author(s) (or their employer(s)) 2020. Re-use permitted under CC BY-NC. No commercial re-use. See rights and permissions. Published by BMJ.

${ }^{1}$ Department of Laboratory, People's Hospital of Yuxi City, Yuxi, Yunnan Province 653100, China

${ }^{2}$ Department of Critical Care Medicine, People's Hospital of Yuxi City, Yuxi, China

${ }^{3}$ Department of Nephrology, People's Hospital of Yuxi City, Yuxi, China

${ }^{4}$ Department of Laboratory, People's Hospital of Yuxi City, Yuxi, China

${ }^{5}$ Department of Laboratory, Sixth Affiliated Hospital of Kunming Medical University, Yuxi, Yunnan, China

Correspondence to

Dr Lei Feng; fngj2004@163.com

\section{ABSTRACT}

Objectives This study aimed to investigate the dynamic trends in total cholesterol (TC), triglyceride (TG), highdensity lipoprotein cholesterol (HDL-C) and low-density lipoprotein cholesterol (LDL-C) levels with ageing.

Design A Chinese population-based cross-sectional study. Setting A physical examination centre of a general hospital.

Participants Adult subjects (178 167: 103461 men and 74706 women) without a known medical history or treatments that affect lipid metabolism.

Main outcome measures Dynamic trends in the above-mentioned lipid parameters with ageing were explored; turning points of age were established using age stratification and validated by fitted multivariate linear regression modelling.

Results Age was found to be an independent factor extensively associated with lipid levels in both sexes when adjusted for serum glucose, body mass index, lifestyle, drinking and smoking. Age was positively associated with TC, logarithm-transformed TG (LnTG) and LDL-C levels in men $\leq 40, \leq 40$ and $\leq 60$ years old (yo) and in women $\leq 60$, $\leq 70$ and $\leq 60$ yo, respectively. Conversely, age correlated negatively with TC, LnTG and LDL-C levels in men $\geq 61$, $\geq 41$ and $\geq 61$ yo and in women $\geq 61, \geq 71$ and $\geq 61$ yo, respectively. TC, TG and LDL-C levels in women were initially lower than those in men but surpassed those in men in $51-55,61-65$ and $51-55$ yo age groups. The trends in HDL-C levels with age were relatively irregular, although HDL-C levels in women were higher than in men for all age groups.

Conclusions The definition of dyslipidaemia, the atherosclerotic cardiovascular disease risk assessment and the initiation/goals of statin therapy should fully consider age-related trends in lipid levels and sex differences.

\section{INTRODUCTION}

Atherosclerotic cardiovascular disease (ASCVD) is highly prevalent in middle-aged men and women, ${ }^{1}$ and hyperlipidaemia has been recognised as a major risk factor. ${ }^{2} 3 \mathrm{An}$ abundance of epidemiological studies and randomised controlled trials have evaluated

\section{Strengths and limitations of this study}

- The uneven distribution of confounders and regression dilution bias were reduced in this large-scale cross-sectional study with 178167 subjects.

- Dynamic trends in lipid levels with ageing were explored by age and sex stratifications.

- Turning points of age positively, invariably and negatively associated with lipid levels were established and validated by fitted multivariate linear regression modelling.

- Age as an independent variable might prevent reverse causation, an inherent defect in crosssectional studies.

- Other shortcomings of a single-centre, crosssectional study could not be avoided in this study.

cholesterol carried by circulating apolipoprotein (apo) B-containing lipoproteins, namely, non-high-density lipoprotein cholesterol (non-HDL-C) and low-density lipoprotein cholesterol (LDL-C), which are root causes of atherosclerosis, reporting that a lower cholesterol level can reduce the incidence of ASCVD. ${ }^{2-4}$

Nonetheless, associations between major lipids and apolipoproteins and the risk of vascular disease have not been reliably quantified, ${ }^{5}$ mainly due to the following issues. First, some ASCVD risk-estimator applications include LDL-C/total cholesterol (TC) as predictors, whereas other risk scores do not include LDL-C/TC as independent variables. ${ }^{6-9}$ Second, although a series of guidelines for the treatment of blood cholesterol levels to reduce ASCVD risk in adults have been provided, ${ }^{2}{ }^{3}$ neither the targets of intervention in dyslipidaemia management nor the treatment goals for non-HLD-C, LDL-C and apoB incorporate age-related trends in lipid levels and sex differences in these trends. The 2013 American College of Cardiology (ACC)/American Heart Association (AHA) guideline identified four 
major groups benefiting from statins (ie, a reduction in ASCVD events with statin therapy). Age stratification is reflected in two primary prevention groups: individuals with diabetes who are 40-75 years old (yo) with LDL-C $70-189 \mathrm{mg} / \mathrm{dL}$ and those without diabetes and with estimated a 10-year ASCVD risk $\geq 7.5 \%$ and who are $40-75$ yo with LDL-C $70-189 \mathrm{mg} / \mathrm{dL}$. ${ }^{3}$ However, no sex difference was included in the key recommendations. ${ }^{3}$ Unique features in cardiovascular disease (CVD) among women are an ongoing concern. ${ }^{10}$ Indeed, in contrast to men, studies indicate that risks for stroke and heart failure through middle and older age in women typically exceed their risk for coronary heart disease (CHD) ${ }^{10}$ Guidelines for CVD prevention in women recommend statin therapy in those $>60$ yo and with an estimated CHD risk $>10 \%$ and high-sensitivity C reactive protein (hsCRP) $>2 \mathrm{mg}$ / $\mathrm{dL}$ after lifestyle modification and no acute inflammatory process and aspirin therapy in women $\geq 65$ yo with prescribed circumstances ${ }^{10}$; however, these age stratifications do not have a very strong theoretical basis. Ridker et al developed the Reynolds Risk Scores to predict global cardiovascular risk for women and men. ${ }^{11}{ }^{12}$ Although the authors concluded that they validated two improved algorithms for assessing global cardiovascular risk in women with high accuracy, the median age (IQR) of derivation and validation cohorts were $52(48-58)$ and 52 (49-59) yo, respectively. ${ }^{11}$ Thus, the participants tended to be older, and only a single coefficient of age $(0.0799$ for the Reynolds Risk Score) was given (indicating a single linear relationship). ${ }^{11}$ In Ridker et $a l$ s prediction model for men, age was treated as three conditions: $<60,60-69$ and $\geq 70$ yo. ${ }^{12}$ These Reynolds Risk Scores did not take into account that age might have a curvilinear relationship with other risk factors, such as blood lipid levels. In the Framingham study, a single multivariable risk function that predicts the risk of developing all CVD and of its constituents was derived from 8491 participants with a mean age of $49 \mathrm{yo}^{13}$; in the algorithms, the regression coefficients of log-transformed age for middle-aged men and women were 2.33 and 3.06, respectively. ${ }^{13}$ Unfortunately, this study also failed to fully consider the pairwise relationship between covariates.

Our understanding of blood lipids has long been mainly limited to their role in the development and progression of ASCVD, whereas we know less about dynamic trends in blood lipid levels over the entire lifespan or differences in these trends between men and women. ${ }^{14}$ In fact, knowledge regarding the age-related dynamic characteristics of blood lipid levels in the general population is a prerequisite for defining dyslipidaemia and for interpreting the relationship between dyslipidaemia and the pathogenesis of ASCVD. In addition, as age and blood lipid levels are two classic risk factors for ASCVD, ${ }^{9}$ determining agerelated trends in lipid levels and rational age stratification in analytical models are essential to improve existing ASCVD risk-estimator applications.

To examine how age is associated with blood lipid levels and whether turning points exist in these associations, dynamic trends in TC, triglyceride (TG), LDL-C and HDL-C levels were explored in 178167 Chinese individuals (103 461 men and 74706 women) in this study.

\section{METHODS}

\section{Patient and public involvement}

No patients or members of the public were involved in the design of this study.

\section{Study design and participants}

To evaluate dynamic trends in TC, TG, LDL-C and HDL-C levels with ageing in the general Chinese population, we designed a cross-sectional study that satisfies the following conditions: (1) a large-scale sample size; (2) a wide age range (18-99 yo); (3) sex stratification; and (4) adjustment for major confounding factors.

A total of 185435 (107594 men and 77841 women) local residents attending an annual physical examination at the People's Hospital of Yuxi City (Yunnan Province, P.R. China) in 2017 were initially enrolled in this study. Subjects who had known familial hyperlipidaemia, hypothyroidism, chronic kidney disease, nephrotic disease, obstructive liver disease, type 1 or type 2 diabetes, or excessive alcohol use or were using lipid-lowering medications, retinoids, cyclosporine, tacrolimus, oestrogen, progestin (including oral contraceptive use) and/or glucocorticoid therapy at the time of the physical examination were excluded from the study. Women who were pregnant or had known polycystic ovary syndrome were also excluded. Ultimately, 178167 subjects (103 461 men and 74706 women) were included in the analyses (figure 1A).

\section{Epidemiological data and medical history}

Medical history, smoking status, level of alcohol consumption and lifestyle factors were collected using a questionnaire. Smoking was defined as smoking 10 or more cigarettes daily for at least 1 year. Moderate alcohol consumption was defined as up to one alcoholic drink per day for women and up to two per day for men. Physical activity was assessed using a questionnaire to record exercise and physical activity. Briefly, an inactive lifestyle was defined as an individual not meeting the following criteria: at least 2 hours and $30 \mathrm{~min}$ a week of moderateintensity aerobic physical activity (brisk walking, dancing, gardening, housework and domestic chores, traditional hunting and gathering, active involvement in games and sports with children or walking domestic animals, general building tasks, and carrying or moving moderate loads), 1 hour and $15 \mathrm{~min}$ a week of vigorous-intensity aerobic physical activity (running, walking or climbing briskly up a hill, fast cycling, aerobics, fast swimming, competitive sports and games, heavy shovelling or digging ditches, and carrying or moving heavy loads) or an equivalent combination of moderate-intensity and vigorous-intensity aerobic activity. For more details, see the 2008 Physical Activity Guidelines for Americans Summary and the definitions for moderate-intensity and vigorous-intensity 
A

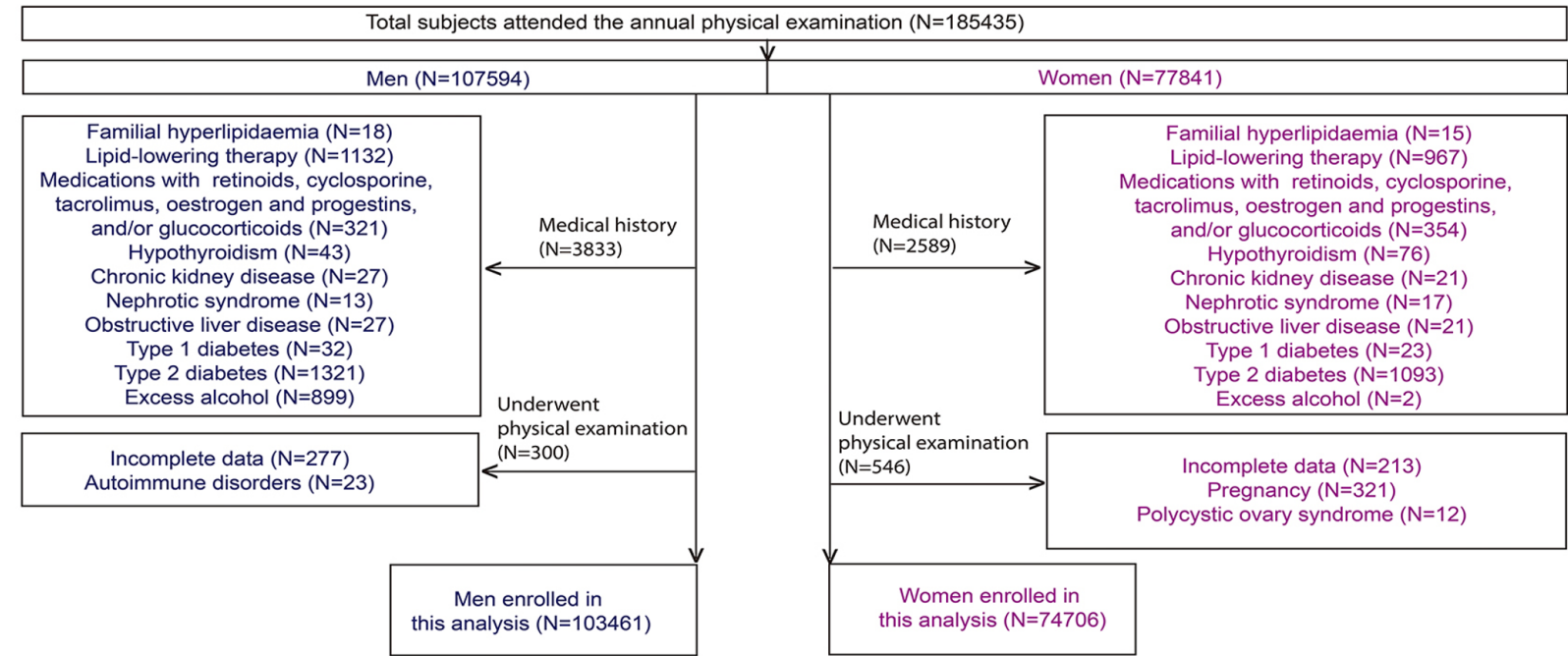

B

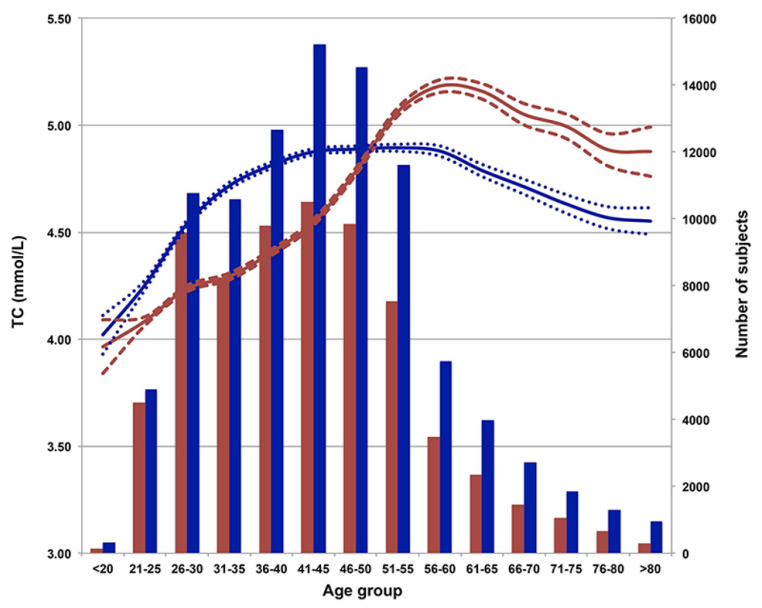

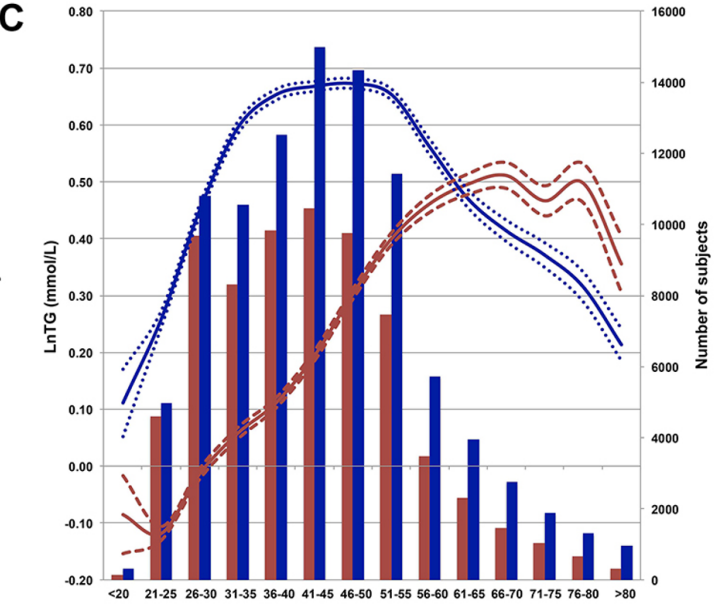

Age group
D

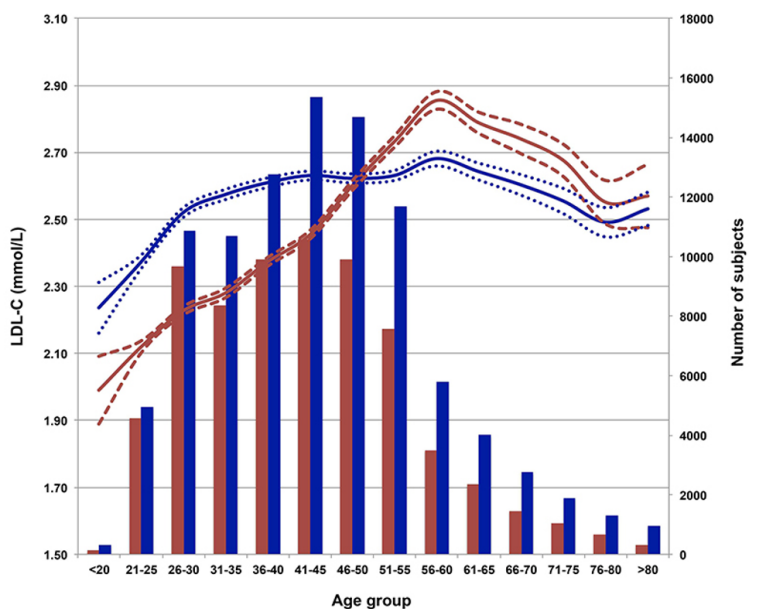

E

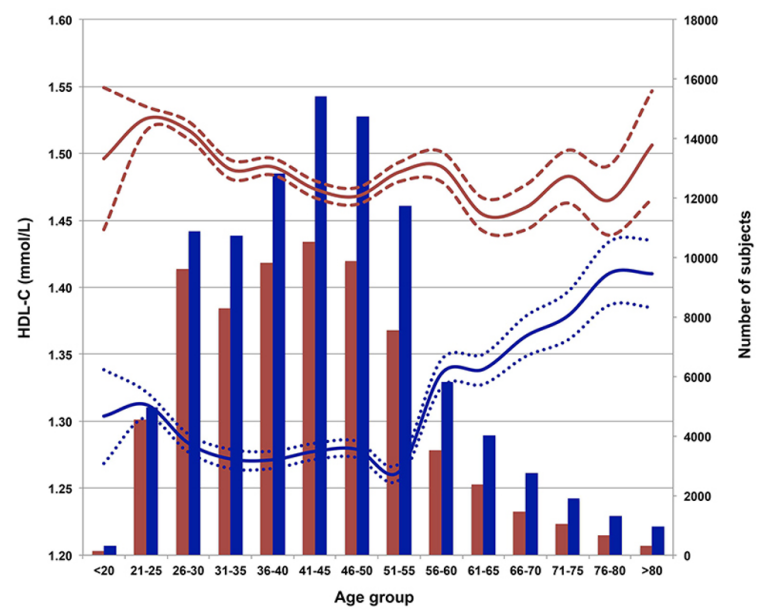

Figure 1 Flow chart of the study participants and lipid levels according to age range. (A) Flow chart of the participants. In the following graphs, $(B)$ to $(E)$, the red and blue lines indicate trends for women and men, respectively, and according to the left ordinates. Dotted lines are the $95 \% \mathrm{Cl}$ of each corresponding mean. Red and blue bars indicate the numbers of women and men in each age range and according to the right ordinates. (B) TC levels plotted by age group. (C) LnTG levels plotted by age group. (D) LDL-C levels plotted by age group. (E) HDL-C levels plotted by age group. HDL-C, high-density lipoprotein cholesterol; LDL-C, low-density lipoprotein cholesterol; TC, total cholesterol; TG, triglyceride. 
physical activity. ${ }^{15} 16$ Body mass index (BMI) was calculated as weight divided by height in metres squared $(\mathrm{kg} /$ $\mathrm{m}^{2}$ ).

\section{Laboratory biochemical parameters}

Laboratory biochemical parameters were measured in our hospital laboratory using routine procedures. Briefly, a blood sample was drawn from the antecubital vein in the morning after overnight fasting. TC, TG, LDL-C and HDL-C levels were directly measured using Vertical Auto Profile Method, an inverted rate-zonal, single-vertical spin, density-gradient ultracentrifugation technique that separates lipoprotein subfractions and measures their contents. All lipid measurements were performed using an ARCHITECT ci16200 Integrated System with the corresponding kits provided by the manufacturer (Abbott, Abbott Park, Illinois, USA). Fasting plasma glucose was assessed using a Roche Modular P chemistry analyser (product \#1876899), GLU reagent kit and Roche Calibrator for Automated Systems (catalogue \#759350) (Roche Diagnostics, Indianapolis, Indiana, USA).

\section{Statistical analysis}

All variables except TG levels generally fit a normal distribution, as assessed by frequency distribution histograms. LnTG displayed a normal distribution after Ln transformation. Therefore, all continuous variables are presented as mean \pm SD.

To explore age-related dynamic trends in TC, TG, LDL-C and HDL-C levels, 5-year intervals were applied to stratify the study subjects, and the means (with 95\% CI) for each lipid were plotted with age stratifications. The turning points of age for changes in lipid level trends were selected using these plots and corresponding analysis of variance. Within each age range, correlations between age and each lipid level were assessed using multivariable linear regression modelling with blood lipids as dependent variables and age as an independent variable. Such fitted, multivariable linear regression modelling validates the necessity and significance of age stratifications and adjusts for any impact of confounding factors. All analyses were performed using SPSS software for Windows (V.18.0; SPSS), and the significance level (alpha) was set at 0.05 .

\section{RESULTS}

\section{General participants' information}

Initially, there were 185435 participants in this study, composed of 107594 male and 77841 female. Of these, 3833 men and 2589 women met the exclusion criteria based on medical history, and 300 men and 546 women were excluded on further examination. Ultimately, data for 103461 men and 74706 women were analysed (figure 1A). The average age of the participants was $42.0 \pm 12.8$ yo for women and $44.9 \pm 13.3$ yo for men. BMI was 22.5 \pm 2.7 (minimum: 15.1; maximum 37.4) for women and $24.7 \pm 2.9 \mathrm{~kg} / \mathrm{m}^{2}$ (minimum: 15.6 ; maximum 40.5) for men. Levels of TC were $4.53 \pm 0.81$ for women
Table 1 General distribution difference of parameters between sexes

\begin{tabular}{llll}
\hline & Female & Male & P value \\
\hline $\begin{array}{l}\text { No. of } \\
\text { subjects }\end{array}$ & 74706 & 103461 & \\
\hline $\begin{array}{l}\text { Age (years } \\
\text { old) }\end{array}$ & $42.0 \pm 12.8$ & $44.9 \pm 13.3$ & $<0.001$ \\
\hline $\begin{array}{l}\text { Smoking } \\
\text { Drinking }\end{array}$ & $1023(1.4 \%)$ & $48734(47.1 \%)$ & $<0.001$ \\
\hline $\begin{array}{l}\text { Inactive } \\
\text { lifestyle }\end{array}$ & $13764(18.4 \%)$ & $20464(19.8 \%)$ & $<0.001$ \\
\hline $\begin{array}{l}\text { BMI (kg/m }) \\
\text { TC (mmol/L) }\end{array}$ & $22.5 \pm 2.7$ & $24.7 \pm 2.9$ & $<0.001$ \\
\hline $\begin{array}{l}\text { LnTG } \\
\text { (mmol/L) }\end{array}$ & $0.19 \pm 0.50$ & $0.57 \pm 0.55$ & $<0.001$ \\
\hline $\begin{array}{l}\mathrm{HDL}-\mathrm{C} \\
\text { (mmol/L) }\end{array}$ & $1.47 \pm 0.33$ & $1.27 \pm 0.29$ & $<0.001$ \\
\hline $\begin{array}{l}\text { LDL-C } \\
\text { (mmol/L) }\end{array}$ & $2.43 \pm 0.71$ & $2.57 \pm 0.77$ & $<0.001$ \\
$\begin{array}{l}\text { Glucose } \\
\text { (mmol/L) }\end{array}$ & $4.88 \pm 0.59$ & $5.02 \pm 0.89$ & $<0.001$ \\
\hline
\end{tabular}

Continuous variables are presented as mean $₫ \pm S D$ and categorical data as number (percentage). Differences between groups were examined by using the t-test or $\chi 2$ test according to the characteristics of the data distribution.

$\mathrm{BMI}$, body mass index; BP, blood pressure; HDL-C, high-density lipoprotein cholesterol; LDL-C, low-density lipoprotein cholesterol; TC, total cholesterol; TG, triglyceride.

and $4.78 \pm 0.87 \mathrm{mmol} / \mathrm{L}$ for men, LnTG $0.19 \pm 0.50$ for women and $0.57 \pm 0.55 \mathrm{mmol} / \mathrm{L}$ for men, HDL-C $1.47 \pm 0.33$ for women and $1.27 \pm 0.29 \mathrm{mmol} / \mathrm{L}$ for men, and LDL-C $2.43 \pm 0.71$ for women and $2.57 \pm 0.77 \mathrm{mmol} / \mathrm{L}$ for men. Glucose levels for all women and men were $4.88 \pm 0.59$ and $5.02 \pm 0.89 \mathrm{mmol} / \mathrm{L}$, respectively, and rates of smoking, drinking and inactive lifestyles for all men and women were $47.1 \%$ and $1.4 \%, 36.6 \%$ and $1.3 \%$, and $19.8 \%$ and $18.4 \%$, respectively (table 1 ).

5-Year interval trends in mean TC, TG, LDL-C and HDL-C levels To assess lifetime trends in lipid levels, 5-year age stratification was applied, and mean lipid levels for each age range were calculated and plotted by sex. Interestingly, non-linear trends between age and lipid levels were found (figure 1B-E).

For men, TC levels increased from $4.02 \pm 0.82 \mathrm{mmol} / \mathrm{L}$ in those $\leq 20$ yo to $4.81 \pm 0.88 \mathrm{mmol} / \mathrm{L}$ in those $36-40$ yo, plateaued between 41 and 60 yo (with mean levels of $4.88-4.89 \mathrm{mmol} / \mathrm{L}$ ), and then decreased from $4.79 \pm 0.91 \mathrm{mmol} / \mathrm{L}$ in those $61-65$ yo to $4.55 \pm 0.97 \mathrm{mmol} / \mathrm{L}$ in those $>80$ yo (figure 1B). The observed trends for TC in women differed from those in men. The increasing TC trend started in the $\leq 20$ yo group $(3.97 \pm 0.71 \mathrm{mmol} / \mathrm{L})$ and terminated in the $56-60$ yo group $(5.18 \pm 0.90 \mathrm{mmol} / \mathrm{L})$, entering a decline phase thereafter (figure 1B). Moreover, the TC levels in women were lower than those in men for 
all age groups younger than 46-50 yo but exceeded those in men after the age of 51-55 yo (figure $1 \mathrm{~B}$ ). The mean TC level of men reached its peak in the 41-45 yo group $(4.88 \pm 0.90 \mathrm{mmol} / \mathrm{L})$, whereas that of women peaked in the $56-60$ yo group $(5.18 \pm 0.90 \mathrm{mmol} / \mathrm{L})$ (figure $1 \mathrm{~B})$.

As shown in figure 1C, LnTG also displayed increasing, plateau and decreasing phases in men $\leq 40$, between 41 and 55 , and $\geq 56$ yo. In women, a relatively regular increasing trend of LnTG was found up to 70 yo. Although LnTG fluctuated after 70 yo, a general downward trend was fitted. The average LnTG was lower in women than in men in the age groups from $\leq 20$ to $56-60$ yo but was higher in women over 61-65 yo.

Regarding LDL-C levels, the trends for men displayed roughly an increasing phase from $2.24 \pm 0.69 \mathrm{mmol} / \mathrm{Lin}$ the $\leq 20$ yo group to $2.68 \pm 0.85 \mathrm{mmol} / \mathrm{L}$ in the $56-60$ yo group, followed by a decreasing phase from $2.64 \pm 0.81 \mathrm{mmol} / \mathrm{L}$ in the $61-65$ yo group to $2.53 \pm 0.78 \mathrm{mmol} / \mathrm{L}$ in the $>80$ yo group (figure 1D). LDL-C levels in women also showed an increasing phase from $1.99 \pm 0.58 \mathrm{mmol} / \mathrm{L}$ in the $\leq 20$ yo group to $2.86 \pm 0.80 \mathrm{mmol} / \mathrm{L}$ in the $56-60$ yo group and then decreased from $2.79 \pm 0.78 \mathrm{mmol} / \mathrm{L}$ in the $61-65$ yo group to $2.57 \pm 0.84 \mathrm{mmol} / \mathrm{L}$ in the $>80$ yo group, with fluctuations (figure 1D). Thus, the time period between 46 and 50 yo is a boundary for LDL-C levels, with levels in women being lower and higher than those in men before and after this age group, respectively.

In contrast, the trends in HDL-C levels were relatively unique, mainly manifesting as irregular trends with age, with women's HDL-C levels being higher than men's for all age groups. Nonetheless, increasing and decreasing phases were also found roughly in men for the $\leq 55$ and $\geq 56$ yo groups and in women for the $\leq 50$ and $\geq 51$ yo groups (figure 1E).

\section{Associations between age and TC levels within age ranges}

To validate the above turning points of age in association with lipid level trends and to adjust for the possible influence of confounding factors on the observed associations, the participants were restratified by age ranges corresponding to increasing, plateau and decreasing phases for each lipid by sex. Univariate analyses of age, glucose levels, BMI, inactive lifestyle, current smoking status and current drinking status were performed, and age, glucose, BMI and inactive lifestyle were found to be possible independent factors associated with lipid levels in men and women. Multivariable linear logic regression modelling was then applied.

As shown in table 2, age was positively and negatively associated with TC levels in men $\leq 40$ yo and $\geq 61$ yo, with coefficients of 0.035 and -0.013 , respectively, but was not associated with TC levels in men 41-60 yo. Serum glucose and inactive lifestyle were common risk factors positively associated with TC levels, regardless of age range. BMI and current drinker status were risk factors for TC levels in men younger than 61 yo. Additionally, being a current smoker was the only risk factor in men aged $41-60$ yo.
Table 2 Factors associated with TC level in age groups by sex

\begin{tabular}{lll}
\hline Variables & B $(95 \% \mathrm{Cl})$ & P value \\
\hline Men $\leq 40$ yo &
\end{tabular}

$n=39$ 241; mean age: $32.0 \pm 5.3$ yo; mean TC: $4.63 \pm 0.88 \mathrm{mmol} / \mathrm{L}$

\begin{tabular}{lll} 
Age, yo & $0.035(0.033 \sim 0.037)$ & $<0.001$ \\
\hline Glucose, mmol/L & $0.217(0.204 \sim 0.230)$ & $<0.001$ \\
BMI, kg/m² & $0.018(0.011 \sim 0.024)$ & $<0.001$ \\
Inactive lifestyle & $0.067(0.061 \sim 0.073)$ & $<0.001$ \\
Current smoker & $/$ & $/$ \\
Current drinker & $0.031(0.028 \sim 0.035)$ & $<0.001$ \\
Men 41-60 yo & &
\end{tabular}

$n=47$ 116; mean age: 48.8 \pm 5.2 yo; mean TC: $4.89 \pm 0.91 \mathrm{mmol} / \mathrm{L}$

\begin{tabular}{lll} 
Age & / & $/$ \\
\hline Glucose & $0.114(0.106 \sim 0.123)$ & $<0.001$ \\
\hline BMI & $0.128(0.120 \sim 0.134)$ & 0.008 \\
\hline Inactive lifestyle & $0.111(0.118 \sim 0.121)$ & $<0.001$ \\
\hline Current smoker & $0.055(0.049 \sim 0.058)$ & $<0.001$ \\
\hline Current drinker & $0.019(0.017 \sim 0.020)$ & $<0.001$ \\
\hline Men $\geq 61$ yo & & \\
n=10 800; mean age: $69.5 \pm 6.9$ yo; mean TC: $4.70 \pm 0.94 m m o l / L$ \\
\hline Age & $-0.013(-0.016 \sim 0.011)$ & $<0.001$ \\
\hline Glucose & $0.052(0.035 \sim 0.068)$ & $<0.001$ \\
\hline BMl & $/$ & $/$ \\
\hline Inactive lifestyle & $0.011(0.009 \sim 0.013)$ & $<0.001$ \\
\hline Current smoker & $/$ & $/$ \\
Current drinker & $/$ & $/$
\end{tabular}

n=63 679; mean age: 40.0 \pm 9.9 yo; mean TC: $4.54 \pm 0.85 \mathrm{mmol} / \mathrm{L}$

\begin{tabular}{lll} 
Age & $0.032(0.031 \sim 0.033)$ & $<0.001$ \\
Glucose & $0.119(0.107 \sim 0.131)$ & $<0.001$ \\
BMI & $0.038(0.018 \sim 0.058)$ & $<0.001$ \\
Inactive lifestyle & $0.132(0.147 \sim 0.212)$ & $<0.001$ \\
Women $\geq 61$ yo & & \\
$n=5831 ;$ mean age: $68.6 \pm 6.4$ yo; mean TC: $5.06 \pm 0.95 \mathrm{mmol} / \mathrm{L}$ \\
\hline Age & $-0.016(-0.020 \sim-0.012)$ & $<0.001$ \\
Glucose & $/$ & $/$ \\
BMI & $/$ & $/$ \\
\hline Inactive lifestyle & $0.103(0.091 \sim 0.124)$ & $<0.001$ \\
\hline
\end{tabular}

$B$ is a coefficient indicating a one-unit increase in an independent variable in multivariable linear logic regression analyses. "/" indicates that a variable was not significantly associated with LDL-C level in a given model.

BMI, body mass index; TC, total cholesterol; yo, years old.

Among women, age was positively and negatively associated with TC levels in those $\leq 60$ and $\geq 61$ yo, with coefficients of 0.032 and -0.016 , respectively. Having an inactive lifestyle was the only factor positively associated with TC levels in women, and glucose and BMI were only positively associated with TC levels in those $\leq 60$ yo. 
Table 3 Factors associated with LnTG in age groups by sex

\begin{tabular}{llc}
\hline Variables & B $(95 \% \mathrm{Cl})$ & P value \\
\hline Men $\leq 40$ yo &
\end{tabular}

n=39 173; mean age: $32.0 \pm 5.3$ yo; mean LnTG: $0.53 \pm 0.57 \mathrm{mmol} / \mathrm{L}$

$\begin{array}{lll}\text { Age } & 0.025(0.024 \sim 0.026) & <0.001 \\ \text { Glucose } & 0.141(0.132 \sim 0.149) & <0.001 \\ \text { BMI } & 0.134(0.118 \sim 0.152) & <0.001 \\ \text { Inactive lifestyle } & / & / \\ \text { Current smoker } & / & / \\ \text { Current drinker } & 0.043(0.056 \sim 0.077) & <0.001 \\ \text { Men 41-55 yo } & & \end{array}$

$n=40$ 763; mean age: $47.5 \pm 4.1$ yo; mean LnTG: $0.67 \pm 0.54 \mathrm{mmol} / \mathrm{L}$

\begin{tabular}{lll} 
Age & $-0.005(-0.003 \sim-0.000)$ & $<0.001$ \\
\hline Glucose & $0.116(0.111 \sim 0.122)$ & $<0.001$ \\
BMI & $0.128(0.106 \sim 0.152)$ & $<0.001$ \\
\hline Inactive lifestyle & $0.107(0.119 \sim 0.213)$ & $<0.001$ \\
Current smoker & $0.098(0.101 \sim 0.131)$ & $<0.001$ \\
Current drinker & $0.032(0.045 \sim 0.078)$ & 0.002 \\
Men $\geq 56$ yo & &
\end{tabular}

$n=16$ 586; mean age: 65.5 \pm 7.8 yo; mean LnTG: $0.45 \pm 0.52 \mathrm{mmol} / \mathrm{L}$

\begin{tabular}{|c|c|c|}
\hline Age & $-0.013(-0.014 \sim-0.012)$ & $<0.001$ \\
\hline Glucose & $0.073(0.066 \sim 0.080)$ & $<0.001$ \\
\hline BMI & $0.106(0.088 \sim 0.126)$ & $<0.001$ \\
\hline Inactive lifestyle & / & / \\
\hline Current smoker & $0.027(0.021 \sim 0.031)$ & $<0.001$ \\
\hline \multicolumn{3}{|l|}{ Current drinker } \\
\hline \multicolumn{3}{|l|}{ Women $\leq 70$ yo } \\
\hline \multicolumn{3}{|c|}{$n=67$ 485; mean age: $41.3 \pm 11.2$ yo; mean LnTG: $0.18 \pm 0.50 \mathrm{mmol} / \mathrm{L}$} \\
\hline Age & $0.016(0.016 \sim 0.016)$ & $<0.001$ \\
\hline Glucose & $0.123(0.116 \sim 0.129)$ & $<0.001$ \\
\hline BMI & $0.101(0.090 \sim 0.110)$ & $<0.001$ \\
\hline Inactive lifestyle & / & / \\
\hline \multicolumn{3}{|l|}{ Women $\geq 71$ yo } \\
\hline \multicolumn{3}{|c|}{$n=2013$; mean age: $76.0 \pm 4.1$ yo; mean LnTG: $0.46 \pm 0.44 \mathrm{mmol} / \mathrm{L}$} \\
\hline Age & $-0.007(-0.012 \sim-0.003)$ & $<0.001$ \\
\hline Glucose & $0.074(0.050 \sim 0.098)$ & $<0.001$ \\
\hline BMI & $0.044(0.018 \sim 0.072)$ & 0.001 \\
\hline Inactive lifestyle & / & / \\
\hline
\end{tabular}

$B$ is a coefficient indicating a one-unit increase in an independent variable in multivariable linear logic regression analyses. "/" indicates that a variable was not significantly associated with LDL-C level in a given model.

BMI, body mass index; LDL-C, low-density lipoprotein cholesterol; TG, triglyceride; yo, years old.

\section{Associations between age and LnTG in age ranges}

As shown in table 3 , age was a factor positively associated with LnTG in men $\leq 40$ yo (coefficient: 0.025 ) but negatively associated in men $\geq 56$ yo (coefficient: -0.013 ) and negatively but slightly associated in men aged $41-55$ yo (coefficient: -0.005). Furthermore, BMI and serum
Table 4 Factors associated with LDL-C level in age groups by sex

\begin{tabular}{lll}
\hline Variables & B $(95 \%$ Cl) & P value \\
\hline Men $\leq 60$ yo & & \\
$n=87$ 152; mean age: & $41.2 \pm 9.9$ yo; mean $L D L-C: ~$ & $59 \pm 0.82 \mathrm{mmol} / \mathrm{L}$ \\
\hline Age & $0.006(0.005 \sim 0.007)$ & $<0.001$ \\
Glucose & $0.045(0.039 \sim 0.052)$ & $<0.001$ \\
BMl & $0.108(0.088 \sim 0.126)$ & $<0.001$ \\
Inactive lifestyle & $0.021(0.011 \sim 0.033)$ & 0.001 \\
Current smoker & $/$ & $/$ \\
Current drinker & $/$ & $/$ \\
\hline Men $\geq 61$ yo & &
\end{tabular}

$n=10$ 903; mean age: 69.5 \pm 6.9 yo; mean $L D L-C: 2.59 \pm 0.82 \mathrm{mmol} / \mathrm{L}$

\begin{tabular}{|c|c|c|}
\hline Age & $-0.008(-0.010 \sim-0.006)$ & $<0.001$ \\
\hline Glucose & $0.045(0.031 \sim 0.059)$ & $<0.001$ \\
\hline BMI & $0.064(0.032 \sim 0.096)$ & $<0.001$ \\
\hline Inactive lifestyle & $0.101(0.097 \sim 0.119)$ & $<0.001$ \\
\hline Current smoker & $0.023(0.017 \sim 0.028)$ & $<0.001$ \\
\hline Current drinker & $0.007(0.004 \sim 0.011)$ & $<0.001$ \\
\hline \multicolumn{3}{|l|}{ Women $\leq 60$ yo } \\
\hline \multicolumn{3}{|c|}{$n=64$ 195; mean age: $40.0 \pm 9.9 y o$; mean $L D L-C: 2.44 \pm 0.72 \mathrm{mmol} / \mathrm{L}$} \\
\hline Age & $0.021(0.020 \sim 0.021)$ & $<0.001$ \\
\hline Glucose & $0.111(0.102 \sim 0.121)$ & $<0.001$ \\
\hline BMI & $0.086(0.068 \sim 0.102)$ & $<0.001$ \\
\hline Inactive lifestyle & / & I \\
\hline \multicolumn{3}{|l|}{ Women $\geq 61$ yo } \\
\hline \multicolumn{3}{|c|}{$n=5849$; mean age: $68.6 \pm 6.4$ yo; mean $L D L-C: 2.72 \pm 0.82 \mathrm{mmol} / \mathrm{L}$} \\
\hline Age & $-0.013(-0.017 \sim-0.010)$ & / \\
\hline Glucose & / & / \\
\hline BMI & $0.056(0.024 \sim 0.088)$ & 0.001 \\
\hline Inactive lifestyle & / & / \\
\hline
\end{tabular}

$B$ is a coefficient indicating a one-unit increase in an independent variable in multivariable linear logic regression analyses. "/" indicates that a variable was not significantly associated with LDL-C level in a given model.

BMI, body mass index; LDL-C, low-density lipoprotein cholesterol ; yo, years old.

glucose levels were positively associated with LnTG levels, irrespective of age. Smoking and drinking were risk factors positively associated with LnTG in men $\geq 40$ and $<56$ yo. However, age was an independent factor positively associated with LnTG levels in women $\leq 70$ yo (coefficient: 0.016 ) and negatively and slightly associated in women $\geq 71$ yo (coefficient: -0.007 ). Regardless of age, BMI and glucose correlated positively with LnTG levels in women.

Associations between age and LDL-C levels within age ranges Age was positively associated with LDL-C levels in men and women $\leq 60 \mathrm{yo}$, with coefficients of 0.006 and 0.021 , respectively, and was negatively associated in men and women $\geq 61$ yo, with coefficients of -0.008 and -0.013 , respectively (table 4 ). The absolute values of the 
Table 5 Factors associated with HDL-C level in age groups by sex

\begin{tabular}{|c|c|c|}
\hline Variables & B $(95 \% \mathrm{Cl})$ & $P$ value \\
\hline \multicolumn{3}{|l|}{ Men $\leq 55$ yo } \\
\hline \multicolumn{3}{|c|}{$n=81$ 657; mean age: $40.0 \pm 9.1$ yo; mean $H D L-C: 1.28 \pm 0.37 \mathrm{mmol} / \mathrm{L}$} \\
\hline Age & $-0.001(-0.001 \sim-0.001)$ & $<0.001$ \\
\hline Glucose & $-0.058(-0.061 \sim-0.055)$ & $<0.001$ \\
\hline BMI & $-0.068(-0.076 \sim-0.062)$ & $<0.001$ \\
\hline Inactive lifestyle & / & / \\
\hline Current smoker & / & / \\
\hline Current drinker & / & / \\
\hline Men $\geq 56$ yo & & \\
\hline
\end{tabular}

$n=16$ 765; mean age: $65.5 \pm 7.8$ yo; mean HDL-C: $1.36 \pm 0.40 \mathrm{mmol} / \mathrm{L}$

\begin{tabular}{lll} 
Age & $0.003(0.002 \sim 0.004)$ & $<0.001$ \\
\hline Glucose & $-0.070(-0.076 \sim 0.065$ & $<0.001$ \\
\hline BMI & $-0.082(-0.094 \sim-0.070$ & $<0.001$ \\
\hline Inactive lifestyle & $/$ & $/$ \\
\hline Current smoker & $/$ & $/$ \\
Current drinker & $/$ & $/$ \\
Women $\leq 50$ yo & & \\
$n=52$ 878; mean age: $37.0 \pm 8.0$ yo; mean HDL-C: $1.49 \pm 0.32 \mathrm{mmol} / \mathrm{L}$ \\
\hline Age & $-0.002(-0.003 \sim 0.002)$ & $<0.001$ \\
Glucose & $-0.075(-0.081 \sim 0.070)$ & $<0.001$ \\
BMI & $-0.074(-0.084 \sim-0.062)$ & $<0.001$ \\
Inactive lifestyle & $/$ & $/$ \\
Women $\geq 51$ yo & &
\end{tabular}

$n=16$ 976; mean age: 59.3 \pm 8.1 yo; mean HDL-C: $1.48 \pm 0.33 \mathrm{mmol} / \mathrm{L}$

$\begin{array}{lll}\text { Age } & \text { / } & \text { / } \\ \text { Glucose } & -0.058(-0.064 \sim 0.051) & <0.001 \\ \text { BMI } & -0.064(-0.074 \sim-0.052) & <0.001 \\ \text { Inactive lifestyle } & / & /\end{array}$

$B$ is a coefficient indicating a one-unit increase in an independent variable in multivariable linear logic regression analyses. "/" indicates that a variable was not significantly associated with LDL-C level in a given model.

BMI, body mass index; HDL-C, high-density lipoprotein cholesterol ; LDL-C, low-density lipoprotein cholesterol ; yo, years old.

coefficients were greater in women than in men. BMI was a factor correlating positively with LDL-C levels, irrespective of sex and age, and glucose level and inactive lifestyle were associated with LDL-C in men, regardless of age. Drinking and smoking were associated with LDL-C levels only in men $\geq 61$ yo, and glucose levels were associated with LDL-C levels in women $\leq 60$ yo.

\section{Associations between age and HDL-C levels}

Overall, the trends in HDL-C levels with age were relatively irregular. Although the data presented in figure $1 \mathrm{E}$ indicate increasing and decreasing phases approximately in men $\leq 55$ and $\geq 56$ yo and in women $\leq 50$ and $\geq 51$ yo, multivariable linear logic regression analyses revealed a different association pattern between age and HDL-C levels (table 5). Indeed, in men, age was found to be an independent factor negatively associated with HDL-C levels in those $\leq 55$ yo and positively in those $\geq 56$ yo. For women, age was negatively associated with HDL-C level in all age ranges. BMI was also negatively associated with HDL-C levels, regardless of sex and age, and glucose was negatively associated with HDL-C levels in women $\leq 50$ yo and in men, regardless of age.

\section{DISCUSSION}

Age is a classic unmodifiable risk factor, and LDL-C is a classic modifiable risk factor for CVD. ${ }^{9}$ The association between dyslipidaemia and ASCVD has been explored for decades at tremendous social and economic costs. Regardless, few studies have evaluated how age is associated with lipid levels in the general population over a wide age range. In general, assessment of pairwise correlations between every two covariates is a key quality control process to ensure the reliability of the widely used logistic regression modelling analysis. For correlations between two covariables (eg, age and lipid level) that fit a curvilinear model, most of the current analytical strategies are not well able to 'adjust' the interaction between the two covariables, and it is impossible to provide accurate correlation coefficients in association with a dependent variable (eg, CVD or CHD). In this study, we aimed to determine age-related trends in lipid levels in the general Chinese population. First, age was found to be an independent risk factor associated with an elevation in TC, TG, LDL-C and HDL-C levels in men $\leq 40, \leq 40, \leq 60$ and $\geq 56$ yo, respectively. Second, age was independently associated with declining TC, TG, LDL-C and HDL-C levels in men $\geq 61, \geq 41, \geq 61$ and $\leq 55$ yo, respectively. Third, age was an independent risk factor associated with an elevation of TC, TG and LDL-C levels in women $\leq 60, \leq 70$ and $\leq 60$ yo, respectively. Fourth, age was independently associated with a decline in TC, TG, LDL-C and HDL-C levels in women $\geq 61, \geq 71$, $\geq 61$ and $\leq 50$ yo, respectively. In summary, age showed completely opposite correlation trends with lipid levels between given age ranges. Therefore, age stratification with a theoretical basis should be implemented in the definition of dyslipidaemia, ASCVD risk assessment and the initiation/goals of statin therapy.

Current knowledge of age-related trends in lipid levels is thought to be clear but is in fact vague. Although most consider that TC, LDL-C and TG levels increase up to middle age and then decrease, ${ }^{14}$ the literature on the correlation between age and lipid levels is limited. For example, data from the US cross-sectional National Health and Nutrition Examination Surveys show a favourable trend in serum lipid levels, whereby levels of TC, TG and LDL-C decreased over 22-42 year periods, among US adults. The purpose of this study was not to examine trends in the relationship between age and lipid profiles but to show the effects of trans-fatty acids, lipid-lowering medications and healthy lifestyle factors 
on changes in lipid profiles. ${ }^{17} 18$ A study of 269 healthy white participants (126 men, 143 women) aged 40-60 years reported a significant, linear age-related increase in TG and curvilinear effects of age on LDL-C and TC in both sexes. ${ }^{19}$ Although this is one of the few papers to demonstrate curvilinear relationships between blood lipids and age, ${ }^{14}$ the authors did not reveal further details about this curvilinear relationship. In another study involving 423 adult white participants, TC and LDL-C levels were found to increase up to middle age and then decrease, ${ }^{20}$ but the sample size was too small to provide reliable evidence of age-related trends in lipid levels. A report from the Framingham study with 2222 men and 2677 women aged 20-79 years showed that among younger age groups, plasma TC levels of each group rose in concert but that levels declined in older individuals. ${ }^{21}$ However, the age interval was too large (14 yo), which would obscure the details of the relationship. In summary, the overall understanding of age-related trends in lipid levels to date includes the following shortcomings. First, few studies provide a lifetime trend of lipid levels with age. Second, no previous study has found reliable and detailed data on age turning points in association with lipid level changes using a large-scale sample size and covering a wide age range. Third, few reports have shown an awareness of sex differences in lipid levels throughout the life span.

According to our data, the starting levels of TC, TG and LDL-C in men are higher than those in women, and men face decades of upward trends of these lipids and lipid proteins in the $\leq 40, \leq 40$ and $\leq 60$ age ranges, respectively. We think these lipid level trends render men more susceptible to ASCVD than women. ${ }^{22}{ }^{23}$ In addition, TC, TG and LDL-C levels in women were observed to be lower than those in men, although these levels in women surpassed those in men for the age groups 51-55, 61-65 and 51-55 yo, respectively. This might be associated with the narrowing gap of risk for ASCVD in postmenopausal women. ${ }^{24}$ It is unclear why the HDL-C trends are unique, and although the lifetime trend was irregular in both sexes, HDL-C levels in women were higher than those in men in all age groups.

We also evaluated associations between glucose, BMI, lifestyle, smoking and drinking and lipid levels and the particular patterns found in men and women within given age ranges. However, we do not discuss these issues in depth here due to space constraints.

Ageing is a fundamental and inherent physiological process characterised by deterioration in every facet of a biological system over time..$^{25} 26$ The absorption, synthesis and metabolism of fat and lipoproteins change with age through complex mechanisms. ${ }^{2728}$ Our data illustrate lifetime trends in lipid levels, which might reflect the effect of the ageing process on lipid metabolism in the general Chinese population. Thus, we suggest that the definition of dyslipidaemia should not only be based on pathophysiological evidence of ASCVD but should also take into full account the impact of age and sex.
This is a cross-sectional study with 185435 participants. The lifetime trends in lipid parameters were explored by 5-year age stratification, and the large sample size ensured that enough data were included for each age range. Agerelated trends in lipid levels according to sex are essential for 'precision medicine', and pragmatic studies focused on lipids are often mistaken for duplication without novelty. However, no existing prospective or interventional study has realised the importance of age and sex; therefore, current recommendations for patient-centred management of dyslipidaemia need to be improved by age and sex stratification.

The limitations of this report include the following. First, lipid metabolism is associated with race/geographic origin, ethnic origin, education background, socioeconomic status, occupational status and religious affiliation, among others. ${ }^{109}$ All participants in this study were Chinese adults. Although we believe that the large-scale sample size ensured the representation of Chinese adults, it is unclear whether our results can be applied to other races. Second, reproductive factors, such as parity and menopause, have been shown to correlate with lipid metabolism in women ${ }^{3031}$; as we did not collect data on parity, its effect on age-related trends in lipid levels cannot be clarified. Third, hsCRP, ${ }^{11} 1232$ genetic factors, ${ }^{3}$ sex hormone status, ${ }^{33} 34$ dietary pattern ${ }^{3}$ and mental stress ${ }^{35}$ have also been found to be associated with changes in lipid metabolism, and our data did not include these factors.

Contributors LF, ZT and SN conceived the study, designed the experiments, supervised the project and wrote the manuscript. YZ, YL, CZ, XB, XL, MW, ZY and ZT performed the experiments, analysed the results and wrote the manuscript. All authors had full access to all of the data in the study and can take responsibility for the integrity of the data and the accuracy of the data analysis. The corresponding authors attest that all listed authors meet authorship criteria and that no others meeting the criteria have been omitted.

Funding This work was supported by the Regional Fund Project of the National Natural Science Foundation of China (Serial number: 81460326), Joint Special Funds from the Yunnan Province Science and Technology Department and Department of Applied Basic Research of Kunming Medical University (Serial number: 2018FE001-081) and the Special Funds for Training High-level Health Technical Talents in Yunnan Province (Serial number: D-201644). The funding bodies had no role in the study design; in the collection, analysis and interpretation of data; in the writing of the report; and in the decision to submit the article for publication.

Competing interests All authors have completed the ICMJE uniform disclosure form (available on request from the corresponding authors) and declare: no support from any organisation for the submitted work; no financial relationships with any organisations that might have an interest in the submitted work in the previous three years, no other relationships or activities that could appear to have influenced the submitted work; no spouses, partners, or children have financial relationship that may be relevant to the submitted work; and no non-financial interests that may be relevant to the submitted work.

Patient consent for publication Not required.

Ethics approval This study was conducted in accordance with the principles of the World Medical Association's Declaration of Helsinki. The study protocol was approved by the Review Board of the People's Hospital of Yuxi City (YNYXH2017-003).

Provenance and peer review Not commissioned; externally peer reviewed.

Data availability statement All data relevant to the study are included in the article or uploaded as supplementary information. Extra data are available by emailing LF. 
Open access This is an open access article distributed in accordance with the Creative Commons Attribution Non Commercial (CC BY-NC 4.0) license, which permits others to distribute, remix, adapt, build upon this work non-commercially, and license their derivative works on different terms, provided the original work is properly cited, appropriate credit is given, any changes made indicated, and the use is non-commercial. See: http://creativecommons.org/licenses/by-nc/4.0/.

ORCID iD

Lei Feng http://orcid.org/0000-0003-2731-1194

\section{REFERENCES}

1 Benjamin EJ, Blaha MJ, Chiuve SE, et al. Heart disease and stroke Statistics-2017 update: a report from the American heart association. Circulation 2017;135:e146-603.

2 Jacobson TA, Ito MK, Maki KC, et al. National lipid association recommendations for patient-centered management of dyslipidemia: part 1--full report. J Clin Lipidol 2015;9:129-69.

3 Stone NJ, Robinson JG, Lichtenstein AH, et al. ACC/AHA guideline on the treatment of blood cholesterol to reduce atherosclerotic cardiovascular risk in adults: a report of the American College of Cardiology/American heart association Task force on practice guidelines. J Am Coll Cardiol 2013;129:2889-934.

4 Expert Dyslipidemia Panel of the International Atherosclerosis Society Panel members. An International Atherosclerosis Society Position Paper: global recommendations for the management of dyslipidemia--full report. J Clin Lipidol 2014;8:29-60.

5 Di Angelantonio E, Sarwar N, Perry P, et al. Major lipids, apolipoproteins, and risk of vascular disease. JAMA 2009;302:1993-2000.

6 Goff DC, Lloyd-Jones DM, Bennett G, et al. 2013 ACC/AHA guideline on the assessment of cardiovascular risk: a report of the American College of Cardiology/American heart association Task force on practice guidelines. Circulation 2014;129:S49-73.

7 Cooney MT, Dudina A, D'Agostino R, et al. Cardiovascular risk-estimation systems in primary prevention: do they differ? do they make a difference? can we see the future? Circulation 2010;122:300-10.

8 Lloyd-Jones DM. Cardiovascular risk prediction: basic concepts, current status, and future directions. Circulation 2010;121:1768-77.

9 Payne RA. Cardiovascular risk. Br J Clin Pharmacol 2012;74:396-410.

10 Mosca L, Benjamin EJ, Berra K, et al. Effectiveness-based guidelines for the prevention of cardiovascular disease in women--2011 update: a guideline from the american heart association. Circulation 2011;123:1243-62.

11 Ridker PM, Buring JE, Rifai N, et al. Development and validation of improved algorithms for the assessment of global cardiovascular risk in women: the Reynolds risk score. JAMA 2007;297:611-9.

12 Ridker PM, Paynter NP, Rifai N, et al. C-Reactive protein and parental history improve global cardiovascular risk prediction: the Reynolds risk score for men. Circulation 2008;118:2243-51.

13 D'Agostino RB, Vasan RS, Pencina MJ, et al. General cardiovascular risk profile for use in primary care: the Framingham heart study. Circulation 2008;117:743-53.

14 Park Y-MM, Sui X, Liu J, et al. The effect of cardiorespiratory fitness on age-related lipids and lipoproteins. J Am Coll Cardiol 2015;65:2091-100.
15 The Office of Disease Prevention and Health Promotion, Office of the Assistant Secretary for Health, Office of the Secretary, U.S. Department of Health and Human Services. 2008 physical activity guidelines for Americans summary. Available: https://health.gov/ paguidelines/2008/summary.aspx [Accessed 11 Dec 2019].

16 World Health Organization. Global strategy on diet, physical activity and health. What is moderate-intensity and Vigorous-intensity physical activity? Available: https://www.who.int/dietphysicalactivity/ physical_activity_intensity/en/ [Accessed 11 Dec 2019].

17 Carroll $\overline{M D}$, Kit BK, Lacher DA, et al. Trends in lipids and lipoproteins in US adults, 1988-2010. JAMA 2012;308:1545-54.

18 Carroll MD, Lacher DA, Sorlie PD, et al. Trends in serum lipids and lipoproteins of adults, 1960-2002. JAMA 2005;294:1773-81.

19 Schubert CM, Rogers NL, Remsberg KE, et al. Lipids, lipoproteins, lifestyle, adiposity and fat-free mass during middle age: the Fels longitudinal study. Int J Obes 2006;30:251-60.

20 Siervogel RM, Wisemandle W, Maynard LM, et al. Serial changes in body composition throughout adulthood and their relationships to changes in lipid and lipoprotein levels. The Fels longitudinal study. Arterioscler Thromb Vasc Biol 1998;18:1759-64.

21 Wilson PW, Anderson KM, Harris T, et al. Determinants of change in total cholesterol and HDL-C with age: the Framingham study. $J$ Gerontol 1994;49:M252-7.

22 Go AS, Mozaffarian D, Roger VL, et al. Heart disease and stroke statistics--2014 update: a report from the American Heart Association. Circulation 2014;129:e28-92.

23 Félix-Redondo FJ, Grau M, Fernández-Bergés D. Cholesterol and cardiovascular disease in the elderly. facts and gaps. Aging Dis 2013;4:154-69.

24 Swiger KJ, Martin SS, Blaha MJ, et al. Narrowing sex differences in lipoprotein cholesterol subclasses following mid-life: the very large database of lipids (VLDL-10B). J Am Heart Assoc 2014;3:e000851.

25 Barzilai N, Huffman DM, Muzumdar RH, et al. The critical role of metabolic pathways in aging. Diabetes 2012;61:1315-22.

26 Solon-Biet SM, McMahon AC, Ballard JWO, et al. The ratio of macronutrients, not caloric intake, dictates cardiometabolic health, aging, and longevity in AD libitum-fed mice. Cell Metab 2014;19:418-30.

27 Pollock RD, Carter S, Velloso CP, et al. An investigation into the relationship between age and physiological function in highly active older adults. J Physiol 2015;593:657-80.

28 Rando TA, Chang HY, Aging CHY. Aging, rejuvenation, and epigenetic reprogramming: resetting the aging clock. Cell 2012;148:46-57.

29 Frank ATH, Zhao B, Jose PO, et al. Racial/Ethnic differences in dyslipidemia patterns. Circulation 2014;129:570-9.

30 Lv H, Yang X, Zhou Y, et al. Parity and serum lipid levels: a crosssectional study in Chinese female adults. Sci Rep 2016;6:33831.

31 Pirnat A, DeRoo LA, Skjærven R, et al. Lipid levels after childbirth and association with number of children: a population-based cohort study. PLoS One 2019;14:e0223602.

32 Silva ITda, Sanches LB, Mello APdeQ, et al. [Impact of C-reactive protein on cardiovascular risk in adolescents]. Arq Bras Cardiol 2010;94:585-91.

33 Kelly DM, Jones TH. Testosterone: a metabolic hormone in health and disease. J Endocrinol 2013;217:R25-45.

34 Liu H-H, Li J-J. Aging and dyslipidemia: a review of potential mechanisms. Ageing Res Rev 2015;19:43-52.

35 Tenk J, Mátrai P, Hegyi P, et al. Perceived stress correlates with visceral obesity and lipid parameters of the metabolic syndrome: a systematic review and meta-analysis. Psychoneuroendocrinology 2018;95:63-73. 